\title{
Blumen und ihre Besucher: Nektarkannen im Regenwalddach - Die eindrucksvolle Vielfalt der Marcgraviaceae
}

Stefan Dressler

\begin{abstract}
The family Marcgraviaceae is native to the New World tropics. Their inflorescences bear variously shaped or open nectar vessels. Depending on how the inflorescences are built, the flowers are coloured or at which time of the day they smell or open, the flowers are pollinated by birds or bats. Diurnal and nocturnal insects and possums also are also legitimate pollinators.
\end{abstract}

\section{Zusammenfassung}

Die kleine Familie der Marcgraviaceae ist in den neuweltlichen Tropen heimisch. In den Blütenständen befinden sich verschieden geformte Nektarbehälter. Je nach Struktur, Färbung, Duft werden die Blüten von Vögeln oder Fledermäusen bestäubt. Aber auch tag- und nachtaktive Insekten sowie Opossums gehören zu den Bestäubern.

\section{Eine fast unbekannte Familie mit außergewöhnlichen Nektarbehältern}

Die relativ kleine Familie der Marcgraviaceae wird nur wenigen bekannt sein, wird sie doch recht selten in Botanischen Gärten gezeigt. Die etwa 160 Arten aus sieben Gattungen kommen nur in den Tropen der Neuen Welt von Süd-Mexiko bis Bolivien und auch auf dem Antillenbogen vor. Sie bewohnen in der Regel feuchte Regen- und Nebelwälder. Meist sind es Lianen, die ihre Blüten im hohen Kronendach der Wälder bilden. So kommt es, dass die Vertreter oft recht ungenügend bekannt sind und auch immer noch neue Arten gefunden werden.

Interessant ist die erstaunliche Vielfalt an Blütenständen: es gibt Trauben, Dolden und auch Ähren. Dazu sind in diesen Blütenständen verschiedenste Nektarbehälter vorhanden, die von extrafloralen Nektarien gefüllt werden. Diese sind keine Sporne innerhalb der Blüten wie z. B. beim Rittersporn oder der Akelei, sondern speziell umgebildete Tragblätter im Blütenstand. Auch die Laubblätter haben kleine Nektardrüsen auf der Blattunterseite. Aus diesen lassen sich die Nektartaschen ableiten, welche oft auffällig gefärbt sind.

\section{Vielfalt der Bestäuber bei den Marcgraviaceae}

Die beeindruckende Diversität im Blütenbereich dieser Familie soll hier vorgestellt werden. $\mathrm{Zu}$ dem werden die sehr punktuellen Beobachtun- gen zum Besucherspektrum kommentiert. Es gibt einige zufällige Beobachtungen und wenige systematische Studien zu den Bestäubern einzelner Vertreter der Marcgraviaceae. Insgesamt lässt sich aber ein erstaunlich breites Spektrum an Blütenbesuchern für diese doch recht kleine Familie konstatieren.

Der Grundtyp des Blütenstandes in der Familie ist die Traube: eine einfache Achse mit gestielten Blüten. Am Blütenstiel befindet sich ein zu einem Nektarbehälter umgebildetes Blatt. Die Gattungen Ruyschia und Souroubea haben recht regelmäßige fünfzählige Blüten, wobei sich Ruyschia durch (noch) recht blattähnliche, nur leicht verdickte oder konkav eingesenkte Nektarblätter auszeichnet (Abb. 1), wohingegen in Souroubea schon tiefe Sporne unter jeder Blüte stehen, die darüber hinaus auch oft noch auffällig gefärbt sind. Hier wird von einigen Arten angenehmer Duft beschrieben (süßlich nach Pfirsich, Zimt, Gardenie oder Ananas), teilweise auch nachts. Die auffälligen Vogelfarben Gelb und Orange-Rot sind hier zu finden, aber auch Arten mit weißlichen Blüten. Es ist also zu vermuten, dass hier tag- und nachtaktive Insekten als legitime Bestäuber angesprochen werden wie z. B. Tag- und Nachtfalter, die aufgrund der Größe und Struktur der Blütenstände auch durchaus gute Pollenvektoren wären. Bei Ruyschia dagegen wird auf wenigen Herbarbelegen berichtet, dass kleine Bienen beobachtet wurden beim Ausbeuten der Nektarblätter. Auch 


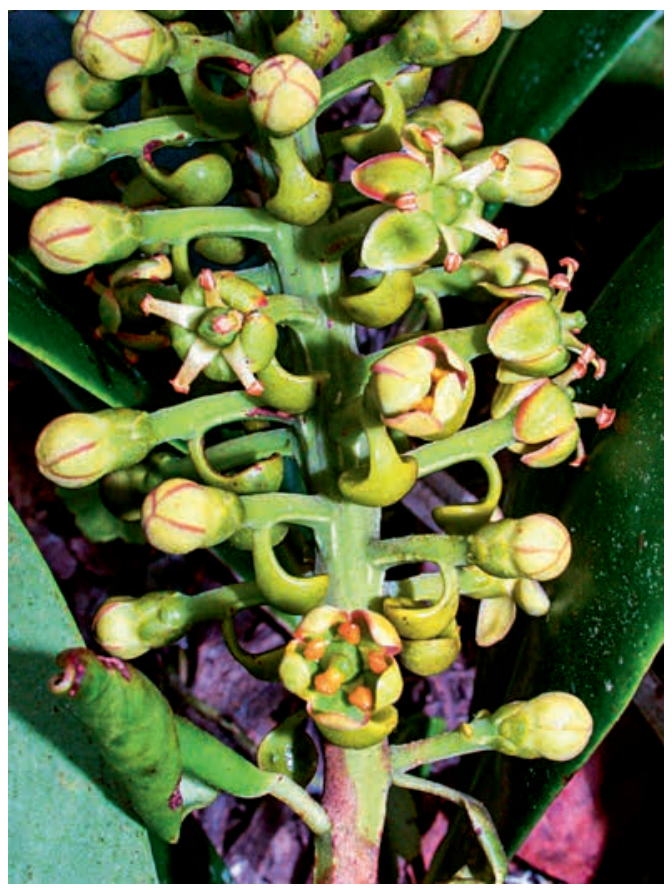

Abb. 1: Ruyschia moralesii aus Costa Rica zeigt traubige Blütenstände mit regelmäßig fünfzähligen Blüten, die jeweils von einem leicht gewölbten oder verdickten Nektarblatt begleitet sind: ein recht simpler Bau, der vielen Besuchern offen steht. (Foto: B. Hammel)

hier wird von Duft berichtet, so dass auch in diesem Fall wohl Insekten die Hauptrolle bei der Bestäubung spielen dürften.

\section{Vogelbeobachtungen an verschiedenen Gattungen der Marcgraviaceae}

Machado \& Lopes (2000) haben in einer längeren Untersuchung über 3 Jahre (insges. 105 Stunden zwischen 4.30 und 24.00 Uhr) an Souroubea guianensis keinen effizienten Bestäuber finden können. Die Blüten öffneten sich zwischen 17.00 und 18.00 Uhr für etwa 6 Tage und dufteten süßlich. Der Pollen wurde in einem Öl präsentiert. Gelegentlicher Kolibribesuch wurde nicht als effektiv angesehen: die Blütenstiele maskieren den Eingang zum Nektarsporn und Kolibris können die Berührung der Blüten (Staubbeutel und Narbe) durch seitlichen Anflug vermeiden. Die Autoren vermuteten Schmetterlingsbestäubung, die sie aber aufgrund der Verarmung im fragmentierten Habitat nicht beobachten konnten. Diesen Bestäubungs-

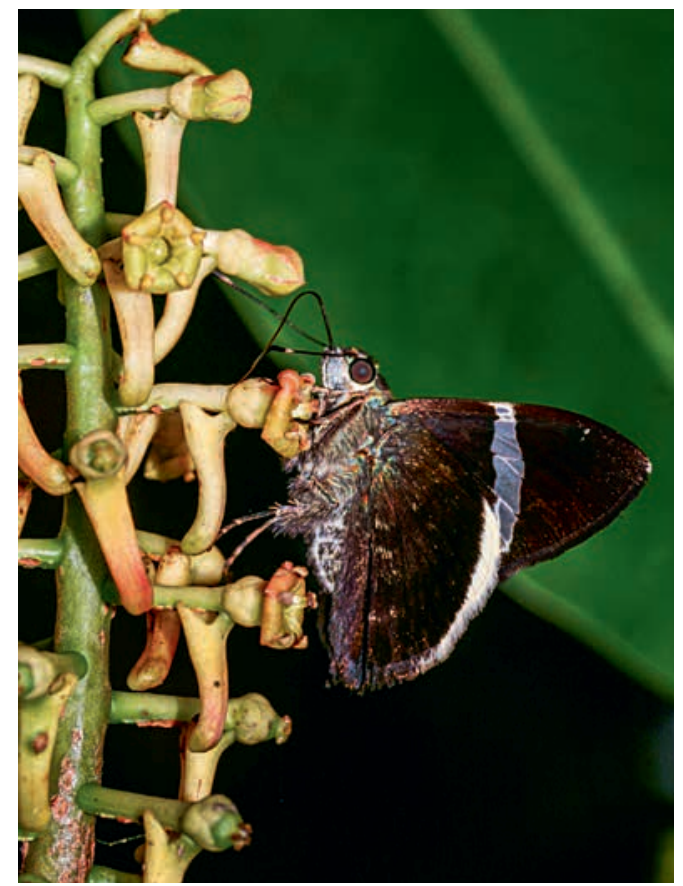

Abb. 2: Souroubea venosa hat schon aufwändigere Nektarsporne, deren Eingang jedoch vom Blütenstiel maskiert wird. Daher kommen nur schmal-rüsselige Schmetterlinge an den Nektar. (Foto: R. MAngelsdorff)

mechanismus konnte Ralph Mangelsdorff an einer anderen Souroubea-Art 2006 in Panama fotografisch dokumentieren (Abb. 2).

Die Gattung Sarcopera ist durch sitzende Blüten an langen Blütenstandsachsen gekennzeichnet, unter denen kleine suppenkellenförmige Nektartaschen sitzen, also durch Ähren als Blütenstandstyp. An einem Vertreter dieser Gattung ist auch erstmalig die Bestäubung durch kletternde Vögel beschrieben worden (Perry 1986). Dies hatte G. Malme bereits 1923 an einer Norantea-Art vermutet. Die Vertreter dieser beiden Gattungen scheinen in der Tat von ihrer Infloreszenzstruktur darauf ausgerichtet zu sein, dass Vögel auf diesen schräg aus dem Kronendach stehenden „Leimruten “ klettern, die Nektarien ausbeuten und mit ihren Füßen den Pollen übertragen (Abb. 3). Die Nektartaschen sind auch in verschiedenen Rottönen gefärbt, was ebenfalls für Vogelbestäubung spricht. 


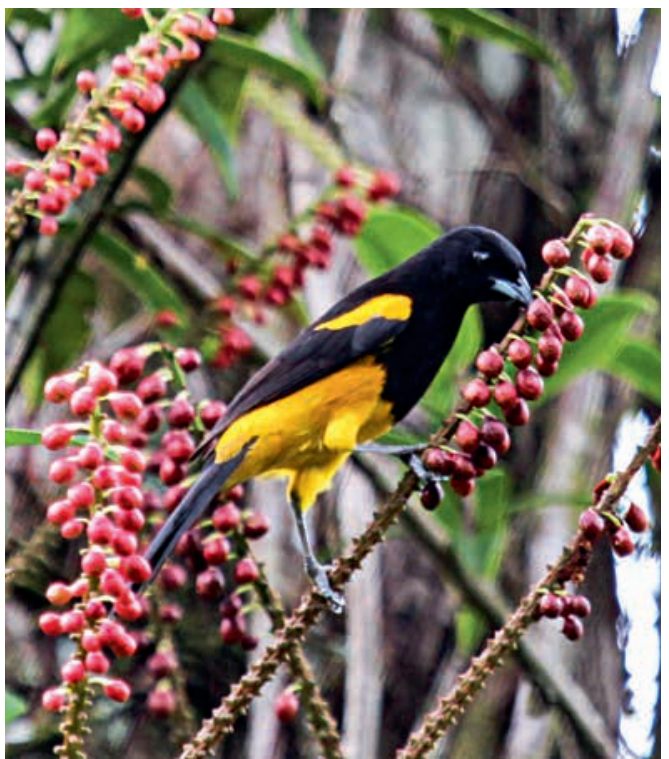

Abb. 3: Sarcopera sessiliflora hat ährenförmige Blütenstände mit unmittelbar an der Achse sitzenden Blüten, die von kletternden Vögeln mit den Füßen bestäubt werden. Hier ein Gelbschultertrupial (Icterus prosthemelas) aus der Familie der Pirole. Natürlich besuchen auch Kolibris diese Blütenstände. (Foto: Ecos Del Bosque)

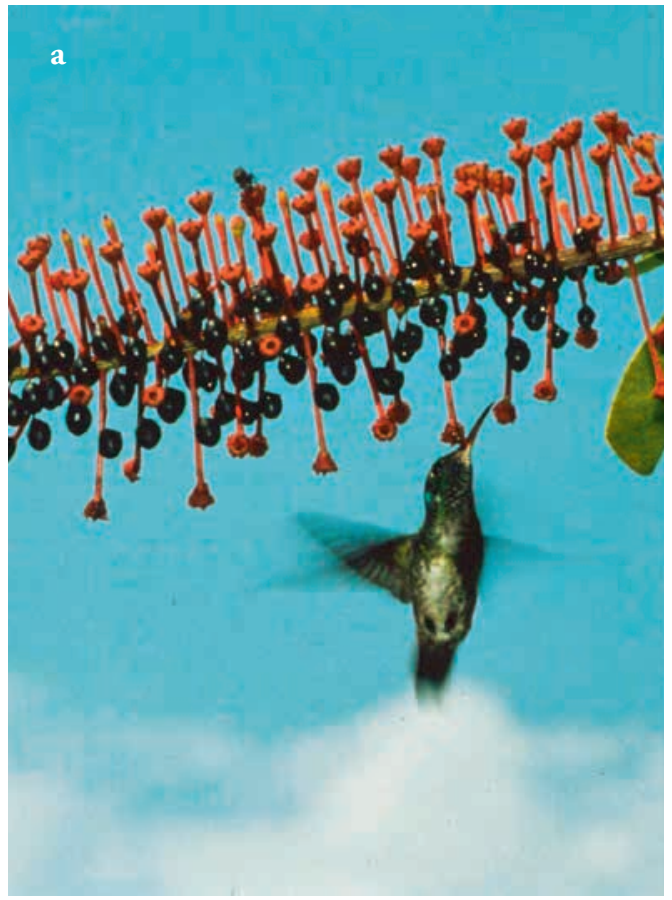

Sazima et al. (1993) publizierten ihre detaillierten Beobachtungen an Schwartzia brasiliensis im atlantischen Küstenregenwald Brasiliens nahe São Paulo. Hier wurden eine Reihe von Vogelarten schwirrend und kletternd an den Blütenständen beobachtet (acht Kolibris, zehn sperlingsartige Vögel). Dabei wurden die Kolibris als ineffiziente Pollenüberträger charakterisiert, weil sie den Nektar ausbeuten, ohne mit den Staubblättern und Narben in Berührung kommen zu müssen (Abb. 4).

\section{Fledermäuse an den Blüten von Marcgraviaceae}

Die übrigen Gattungen zeigen (bis auf Schwartzia brasiliensis) meist wenig Rot oder Orange im Blütenstand. Es sind hier eher grünlich-bräunliche oder auch gelblich-grüne Farbtöne zu finden (Abb. 5). Auch wird hier oft von nächtlichem Erblühen berichtet. Dies zusammen mit dem HellDunkel-Kontrast und mitunter auch Flagelliflorie (das peitschenartige Heraushängen der Blütenstände aus dem Kronendach) deutet auf Fledermäuse als legitime Bestäuber hin.

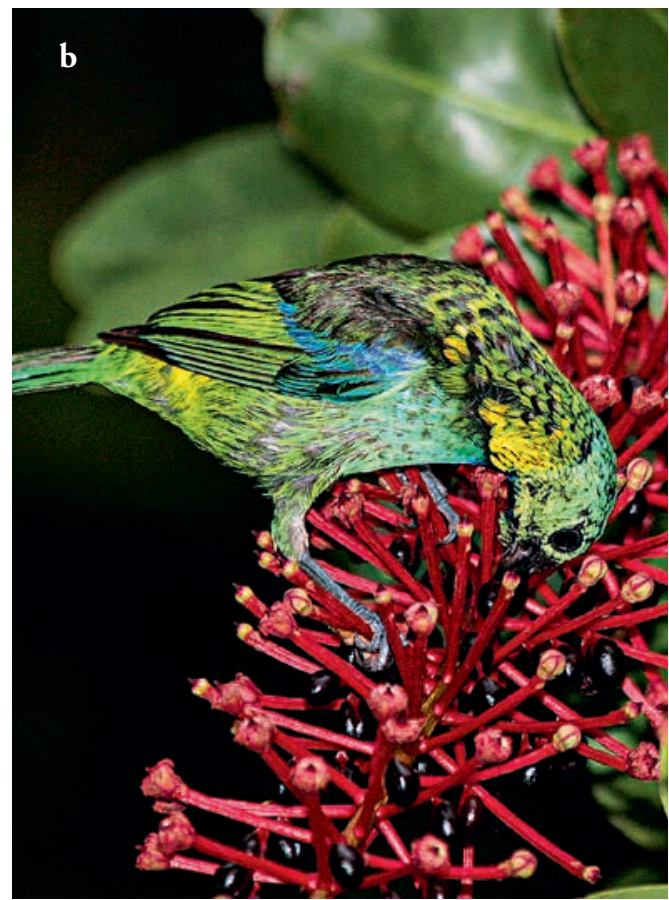

Abb. 4: Schwartzia brasiliensis. (a) Kolibris beuten zwar die Nektartaschen aus, berühren die Blüten (Staubblätter und Narben) aber nur wenig; (b) kletternde Vögel wie hier eine Tangare sind wesentlich „bessere“ Pollenüberträger. (Foto: M. \& I. SAzIMA) 


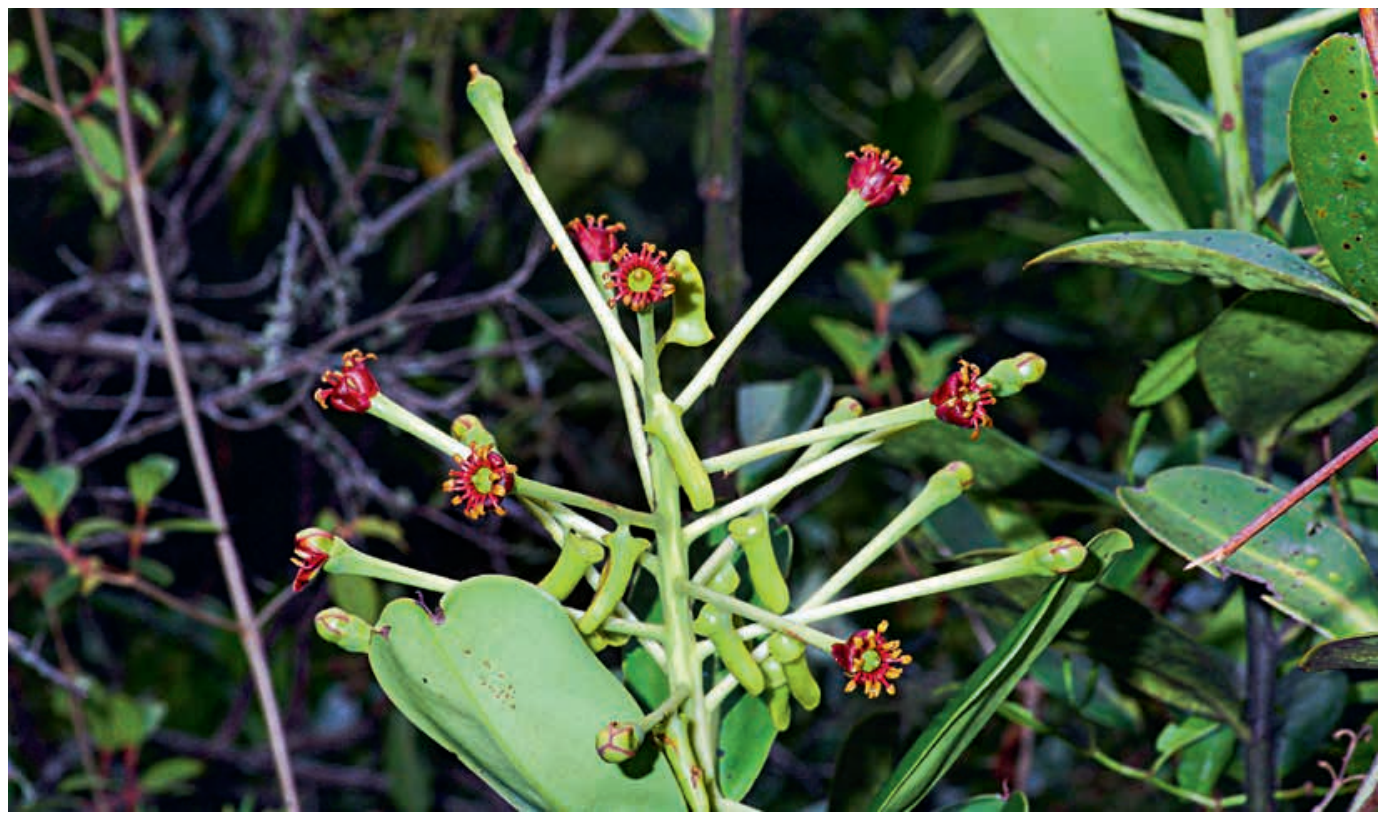

Abb. 5: Schwartzia magnifica aus Peru zeigt nur einen Hell-Dunkel-Kontrast und keine auffälligen Vogelfarben. Das spricht eher für nachtaktive Blütenbesucher. (Foto: G. GERLACH)

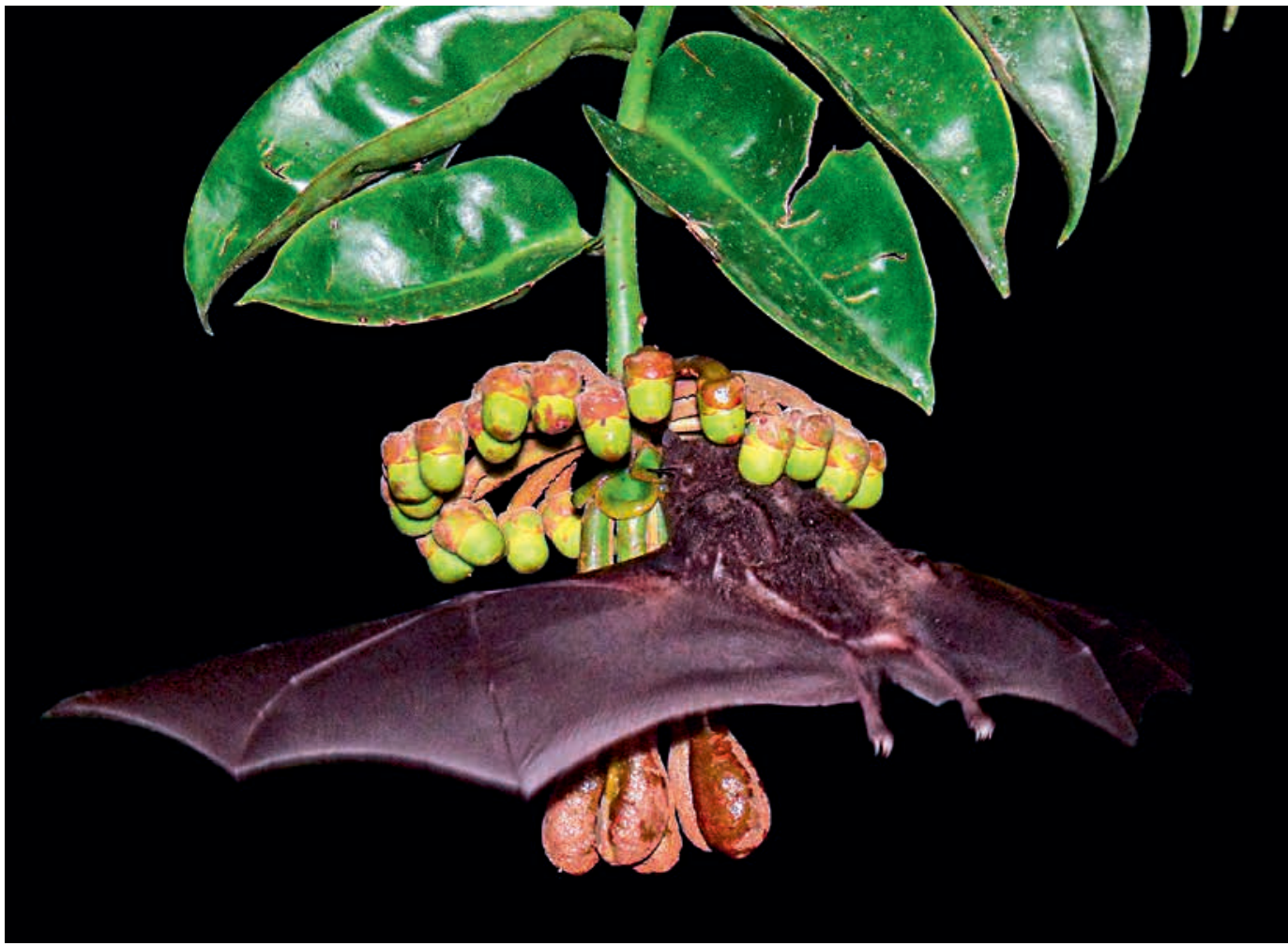

Abb. 6: Marcgravia brownei. (Foto: N. Muchinala) 


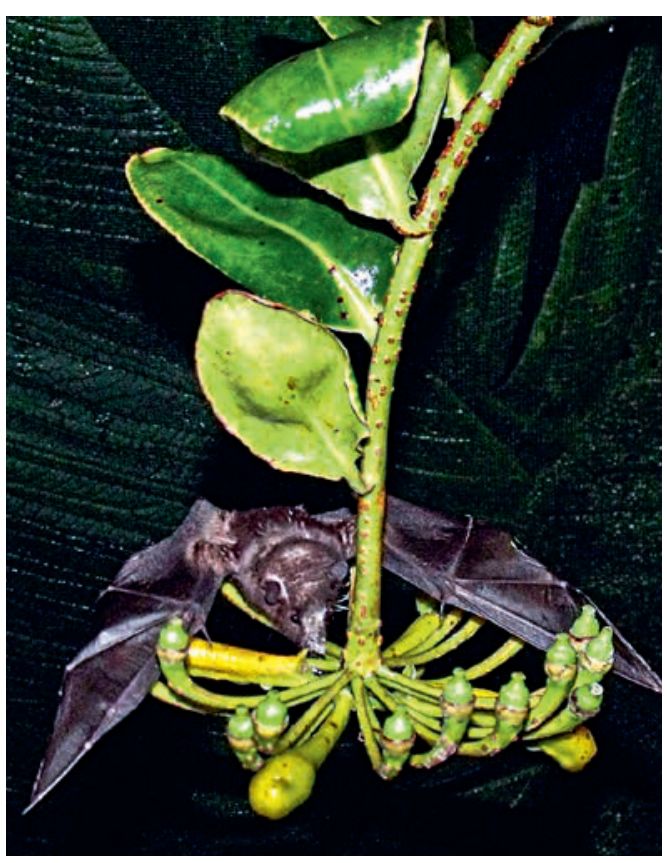

Abb. 7: Marcgravia williamsii. (Foto: N. Muchhala)

Auch haben wir hier eine große Anzahl von Staubblättern, so dass Pinselblumen entstehen (Abb. 8). Oft sind die Blüten groß und robust. Und in der Tat gibt es einige sehr detaillierte Untersuchungen (Tschapka \& von Helversen 1999, Sazima et al. 1999, Muchhala \& JarrinV. 2002), die verschiedene Blumen-Fledermäuse (Glossophaginae) an unterschiedlichen Marcgravia-Arten (Abb. 6-9) beobachtet haben. An einer Art wurden auch Oppossums als legitime Be-

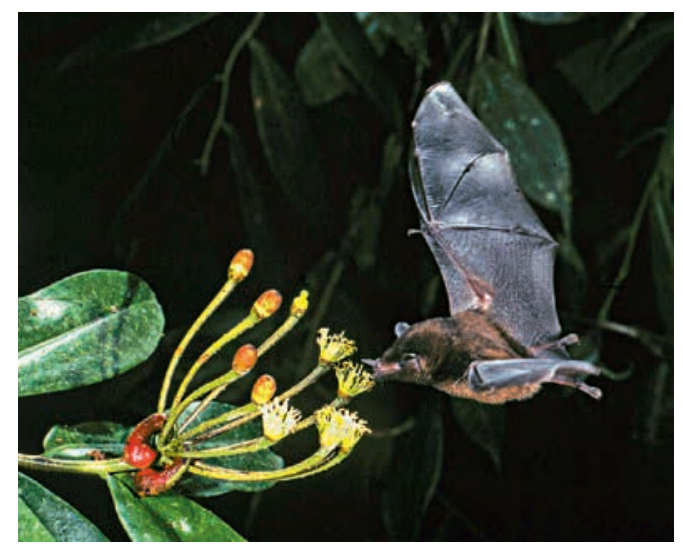

Abb. 8: Marcgravia serrae. (Foto: M. TschapKA) stäuber identifiziert (TsCHAPKa \& von HelverSEN 1999). Dazu kommt die Eigenschaft, dass die Blüten spätnachmittags erblühen und am nächsten Morgen die Staubblätter bereits abgefallen sind.

Ralph Simon und Kollegen untersuchten eine spezielle Marcgravia-Art in Ost-Kuba und fanden eine weitere Auffälligkeit: das letzte Blatt vor dem doldenförmigen Blütenstand ist oft konkav gebogen und ähnelt so einem Parabolspiegel. Durch intensive Beobachtungen und auch Experimente im Flugkäfig konnten sie nachweisen, dass dieses Blatt eine zusätzliche Attraktion bei der Fern-Orientierung der Fledermäuse durch Echolot darstellt (Simon \& al. 2011). Die Fledermäuse lernen, diese Blätter zu erkennen und dann die Nektarquelle unter den Blüten auszubeuten (Abb.10).

Interessanterweise wurde an einer MarcgraviaArt bereits sehr früh Kolibri-Besuch beobachtet und postuliert, dass die Blütenstände ein hervorragendes Beispiel für eine Anpassung an Kolibris als Bestäuber sind (Belt 1874). Die Kolibris führen ihre Schnäbel in die unter dem Blütenkranz hängenden Nektartaschen und bestäuben dabei mit den Flügeln, Köpfen oder Rücken die Pinselblumen über ihnen. Dies ist jedoch sicher nicht der allgemeine Fall: Kolibris probieren jegliche Nektarquelle und werden, da tagaktiv, dabei auch an vielen Pflanzen beobachtet. Nachtaktive Besucher wurden erst viel später überhaupt als potenzielle Bestäuber in Betracht gezogen, weil

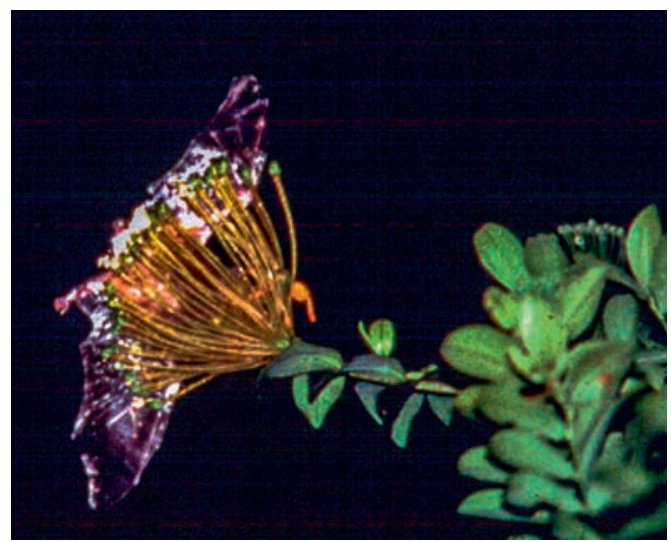

Abb. 9: Marcgravia polyantha. (Foto: M. SAzima) 
man bis weit ins 20. Jahrhundert hinein nachts keine Beobachtungen machen konnte (fehlende Beleuchtungsmöglichkeiten). Es kristallisiert sich also inzwischen heraus, dass Kolibris zwar wirklich auch mitunter bestäuben, aber sicherlich nicht die Haupt- oder typischen Bestäuber sind. Das sind bei den Marcgraviaceae tag- und nachtaktive Insekten (Bienen, Schmetterlinge), kletternde Vögel, Blumenfledermäuse und Opossums. Gelegentliche Blütenbesuche anderer Tiere wie z. B. Anolis oder Primaten werden ebenfalls beobachtet. Die sind aber sicher auf die mehr oder weniger großen Nektarressourcen und eventuell davon angelockte Insekten aus, als dass sie legitime Bestäuber darstellen. Die Pflanzen produzieren oft über einen längeren Zeitraum (z. B. eine Woche) reichlich Nektar (Pinheiro et al. 1995, TschapkA \& v. Helversen 1999), der natürlich alle möglichen Besucher anlockt. Und da die Pflanzen diese Nektarproduktion nicht an- und abschalten können, ist diese Quelle für viele Besucher eine höchst attraktive Ressource. Entscheidend für das Erkennen des oder der legitimen Bestäuber(s) ist der Aufblühzeitpunkt der Blüten und die Mechanik des Anflugs und der Nektarausbeutung innerhalb des Blütenstandes (Tschapka et al. 2006). Weitere detaillierte Feldstudien werden gebraucht.

\section{Literatur}

BeLt, T. 1874: The naturalist in Nicaragua. - London.

Dressler, S. \& Tschapka, M. 2002: Bird versus bat-pollination in the genus Marcgravia and the description of a new species. - Curtis’s Bot. Mag., ser. 6, 19(2): 104-114.

Machado, I.C. \& Lopes, A.V. 2000: Souroubea guianensis Aubl.: Quest for its legitimate pollinator and the first record of tapetal oil in the Marcgraviaceae. Ann. Bot. 85: 705-711.

Malme, G.O.A. 1923: Kolibris als Einbrecher. Bot. Not. (1923): 447-450.

Muchhalla, N. \& Jarrin-V., P. 2002: Flower visitation by bats in cloud forests of western Ecuador. - Biotropica 34: 387-395.

Perry, D. 1986: Expedition in die Anderswelt. Das Kronendach des Regenwaldes erweist sich als Schatzkammer der Wissenschaft. - Geo 1986(2): 46-68.

Pinheiro, M.C.B., Texeira Ormond, W., Alves de Lima, H. \& Rodrigues Correia, M. 1995: Biologia da reprodução de Norantea brasiliensis Choisy (Marcgraviaceae). Rev. Brasil. Biol. 55 (supl. 1): 79-88.

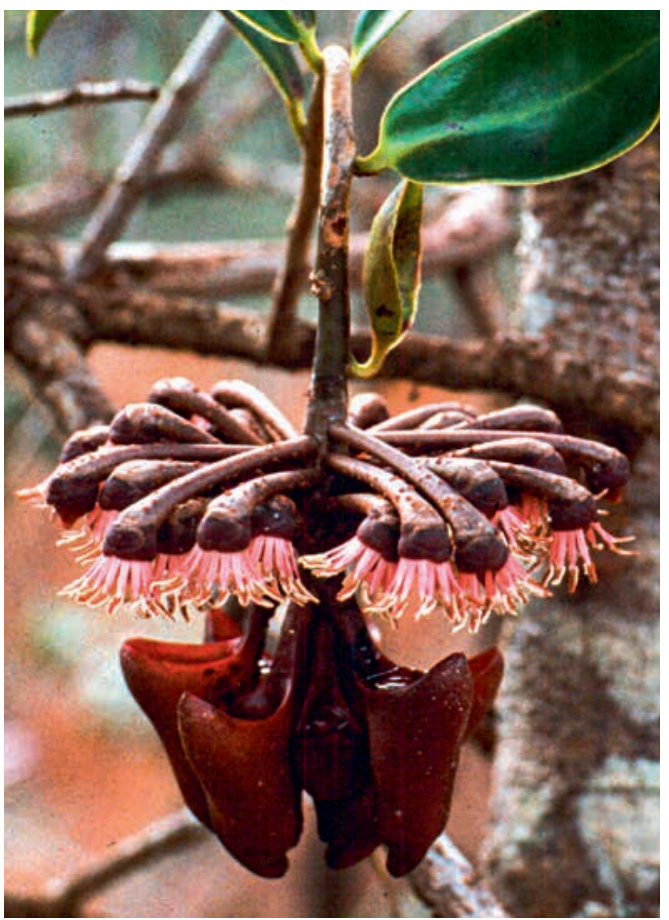

Abb. 10: Marcgravia evenia aus Ost-Kuba zeigt unmittelbar über der Infloreszenz ein bis mehrere parabolspiegelartige Blätter, die zur Fernanlockung von Fledermäusen dienen. (Foto: S. Dressler)

Sazima, I., Buzato, S. \& Sazima, M. 1993. The bizarre inflorescence of Norantea brasiliensis (Marcgraviaceae): Visits of hovering and perching birds. - Bot. Acta 106: 507-513.

Sazima, M., Buzato, S., Sazima, I. 1999: Bat-pollinated Flower Assemblages and Bat Visitors at Two Atlantic Forest Sites in Brazil. - Ann. Bot. 83: 705-712.

Simon, R., Holderied, M.W., Koch, C.U., \& v. Helversen, O. 2011: Floral acoustics: Conspicuous echoes of a dish-shaped leaf attract bat pollinators. - Science 333: 631633. DOI: $10.1126 /$ science. 1204210

Tschapka, M., Dressler, S., \& v. Helversen, O. 2006: Bat visits to Marcgravia pittieri and notes on the inflorescence diversity within the genus Marcgravia (Marcgraviaceae). Flora 201: 383-388.

Tschapka, M. \& v. Helversen, O. 1999: Pollinators of syntopic Marcgravia species in Costa Rican lowland rain forest: bats and opossums. - Plant Biol. 1: 382-388.

\section{Anschrift des Autors}

Dr. Stefan Dressler, Forschungsinstitut Senckenberg, Senckenberganlage 25, 60325 Frankfurt am Main E-Mail: stefan.dressler@senckenberg.de 